\title{
MicroRNA-136-3p inhibits glioma tumorigenesis in vitro and in vivo by targeting KLF7
}

Yanwu Xu

\begin{abstract}
Background: Malignant brain tumors have been a serious threat to human health worldwide. This study aims to investigate the role of miR-136-3p in glioma development.

Methods: Hematoxylin-eosin staining (H\&E) staining was used to determine the pathologic alterations of glioma tissues. Quantitative real-time PCR (qRT-PCR) analysis and GEO2R analysis was performed to examine the expression of miRNAs and genes. Western blot was applied to detect the protein expression. Cell counting kit-8 (CCK-8) and colony formation were used to analyze the glioma cell growth. Trans-well assay was used to determine the cell migration. Annexin V-FITC/PI staining was conducted to determine the cell apoptosis of transfected glioma cells. The dual-luciferase reporter assay was carried out to confirm the binding sites of miR-136-3p on 3' untranslated regions ( $3^{\prime}$ UTR) of Kruppel-like factor 7 (KLF7). Tumor-bearing experiment in nude mice was performed to comprehensively investigate the role of miR-136-3p/KLF7 axis in gliomas.
\end{abstract}

Results: Firstly, the results showed that miR-136-3p was decreased in glioma tissues compared with adjacent tissues. Overexpression of miR-136-3p significantly inhibited cell growth of LN-229 and U251 by decreasing expression of Cyclin A1 and PCNA (proliferating cell nuclear antigen), and it suppressed glioma cell migration by downregulating $\mathrm{N}$-cadherin and elevating E-cadherin levels, and it also promotes glioma cell apoptosis by promoting Bcl2-associated X (Bax) expression but suppressing Bcl-2 expression. Furthermore, we observed that KLF7 was a direct target of miR-136-3p, and KLF7 was negatively regulated by miR-136-3p in glioma cells. Finally, overexpression of KLF7 partly blocked miR-136-3p-induced inhibition of tumor growth in vitro and in vivo.

Conclusions: Targeting miR-136-3p/KLF7 axis might be a novel manner to counter against gliomas.

Keywords: miR-136-3p, KLF7, Glioma, Xenograft tumor model

\section{Background}

Glioma presents one of the most common malignant cancers [1, 2]. Although multiple therapies, including surgery, radiotherapy, and chemotherapy, have been developed for therapeutic treatment of glioma, the prognosis remains poor and the survival rate of 5 years no more than $10 \%$ due to its heterogeneous characteristics $[1,3]$.

Correspondence: ywxu2019shandong@sina.com

Neurosurgery Department, People's Hospital of Lanling County, Linyi, Shandong province 277700 , China
Up to now, few biological risk factors and effective therapeutic targets have been identified to better glioma treatment [4-6]. Thus, it is vital to find novel prognostic and therapeutic targets for glioma patients.

MicroRNAs (miRNAs) are small non-coding RNAs with length of 18-22 nucleotides. It has been reported that binding to $3^{\prime}$ untranslated region ( $3^{\prime}$ UTR) of targeted mRNA is a chief manner for miRNAs exerting their function in diseases [7-9]. Increasing evidences support that a series of miRNAs acts as an essential role

(c) The Author(s). 2020 Open Access This article is licensed under a Creative Commons Attribution 4.0 International License, which permits use, sharing, adaptation, distribution and reproduction in any medium or format, as long as you give appropriate credit to the original author(s) and the source, provide a link to the Creative Commons licence, and indicate if changes were made. The images or other third party material in this article are included in the article's Creative Commons licence, unless indicated otherwise in a credit line to the material. If material is not included in the article's Creative Commons licence and your intended use is not permitted by statutory regulation or exceeds the permitted use, you will need to obtain permission directly from the copyright holder. To view a copy of this licence, visit http://creativecommons.org/licenses/by/4.0/ The Creative Commons Public Domain Dedication waiver (http://creativecommons.org/publicdomain/zero/1.0/) applies to the data made available in this article, unless otherwise stated in a credit line to the data. 
in tumorigenesis including cell proliferation, migration, apoptosis, and angiogenesis [10-13]. For example, miR622 levels predict poor prognosis and it exhibits functions by targeting zinc finger E-box binding homeobox 2 (ZEB2) in glioma [6]. MiR-532-5p inhibits glioma cell proliferation by targeting colony-stimulating factor 1 (CSF1) [14]. MiR-199a suppresses tumor growth and ameliorates chemoresistance via targeting K-RAS via protein kinase B $\alpha$ (namely AKT1) and extracellularregulated protein kinases (ERK) pathways [15]. In this study, we selected to investigate the role of miR-136-3p in gliomas by performing GEO2R analysis of microarray GSE103228, and this bioinformatic data showed that miR-136-3p was decreased in glioma tissues. In the previous studies, miR-136-3p inhibits cell growth and metastasis by targeting solute carrier family 7 member 5 (SLC7A5) and ADAM metallopeptidase domain 9 (ADAM9) in thyroid cancer [16], suggesting that miR-136-3p might function as a tumor suppressor in cancers including gliomas. Similarly, miR-136-5p could enhance glioma cell apoptosis via targeting MTDH (Metadherin) and Bcl-2 [17]. Based on the previous studies, we can conclude that the role of miR136-3p remains unknown in gliomas, and its roles in gliomas need to be further investigated.

To explore the potential molecular mechanisms of miR-136$3 p$ in gliomas, we predicted its targets using TargetScan database. Integration of this bioinformatic analysis with the previous studies, we observed that KLF7 was a direct target of miR-136$3 \mathrm{p}$ in gliomas. In the previous studies, KLF3, targeted by miR450b-3p and miR-185, often severs as an oncogene in gastric cancer [18] and non-small cell lung cancer respectively [19]. And KLF7, regulated by Linc00669/miR-193a axis, may also promote progression of non-small cell lung cancer [20]. Importantly, KLF7 can enhance glioma progression by transcriptionally activating argininosuccinate lyase [21]. Consequently, we can conclude that KLF7 is an oncogene in cancers, such as glioma. Herein, we investigated the role of miR-136-3p/KLF7 axis in glioma cell proliferation, migration, and apoptosis, and we also explored the potential molecular mechanisms. The findings showed that overexpression of miR-136-3p might provide a novel therapeutic target for glioma development.

\section{Methods and materials}

\section{Collection of cancer tissues}

In this study, adjacent tissues and glioma tissues were collected from 41 patients in Shandong Provincial Lanling People's Hospital. The clinical processes were approved by the ethics committee of this Hospital. None of the patients had received treatment before surgery. These collected tissues were put in liquid nitrogen.

\section{Hematoxylin-eosin (H\&E) staining}

We fixed the adjacent tissues and glioma tissues in $4 \%$ paraformaldehyde (Sigma, Shanghai, China). We stained the clinical tissues with hematoxylin and eosin (Sigma) as described previously [1].

\section{Cell culture}

The U251 and LN-229 were bought from ATCC (American Type Culture Collection, Manassas, VA, USA), and they were cultured in Dulbecco's Modified Eagle Medium (DMEM) basic media (Thermo Fisher Scientific, Waltham, Massachusetts, USA), and the media contained $10 \%$ fetal bovine serum, and $100 \mathrm{mg} /$ $\mathrm{mL}$ streptomycin, and $100 \mathrm{U} / \mathrm{mL}$ penicillin (Thermo Fisher Scientific). They were cultured in a $37^{\circ} \mathrm{C}$ sterile incubator with $5 \% \mathrm{CO}_{2}$.

\section{Plasmids, small interfering RNAs, and lenti-viruses}

All the plasmids and RNA fragments were purchased from Sangon (Shanghai, China). These DNAs or RNAs were transfected into glioma cells with Lipofectamine 3000 (Thermo Fisher Scientific). The pcDNA3.1 or lenti-vector was used as an empty vector, and pcDNAKLF7 plasmid, as well as lenti-KLF7, contained a full length of KLF7 complementary DNA, were employed to overexpress KLF7 in glioma cells. MiR-NC (5'-UCU CCG AAC GUG UCA CGU U-3') or lenti-miR-NC was applied as a negative control, and miR-136-3p mimic (5'-CAU CAU CGU CUC AAA UGA GUC U-3') or lenti-miR-136-3p was used to overexpress miR-136-3p, and anti-miR-136-3p (5' -AGA CUC AUU UGA GAC GAU GAU G-3') was applied to knock miR-136-3p down. On the other hand, pmirGLO-KLF7-3' untranslated region (UTR)-WT (wide type) or -Mut (mutant) were constructed by Sangon used for determining the interaction between miR-136-3p and KLF7, and mutant binding sites were synthesized by Sangon.

\section{Quantitative real-time PCR}

We extracted total RNA using TRIzol reagent (Thermo Fisher Scientific). The purified RNA $(1 \mu \mathrm{g})$ was used to produce complementary DNA (cDNA) using a kit for reverse transcription (Thermo Fisher Scientific). Then, SYBR Green Kit, from Thermo Fisher Scientific, was used to determine the expression of miRNAs and mRNAs. The U6 or $\beta$-actin was considered as an internal control of miR-136$3 p$ or mRNAs, respectively. The primers were shown below. Cyclin A1: forward, 5 '-CTC TTA ACC GCG ATC CTC C-3', reverse, 5'-CCA TCC CAA GTG ACG AGC A-3'. PCNA: forward, $5^{\prime}$-CTC TTC CCT TAC GCA AGT CTC A-3', reverse, 5'-GTG CCT CCA ACA CCT TCT TG-3'. E-cadherin: forward, 5'-AAC AGC AAA GGG CTT GGA TTT TG-3', reverse, 5'-CAG CCA GTT GGC AGT GTC TC-3'. N-cadherin: forward, 5'-TGC ATG AAG GAC AGC CTC TT-3', reverse, 5' ${ }^{\prime}$ TGG GTC TCT TTG TCT TGG GC-3'. Bax: forward, 5'-GGG TTG TCG CCC TTT TCT AC-3', reverse, 5'-AGT CGC TTC AGT 
GAC TCG G-3'. Bcl-2: forward, 5'-ATG CGG CCT CTG TTT GAT TTC-3', reverse, 5'-GCA GGC ATG TTG ACT TCA CTT-3'. KLF7: forward, 5' - -3', reverse, 5' - -3'. $\beta$-actin: forward, 5'-GCT CAC CAT GGA TGA TGA TAT CGC-3', reverse, 5'-TAG GAA TCC TTC TGA CCC ATG CC-3'. MiR-136-3p: forward, 5'-CAU CAU CGU CUC AAA U-3', reverse, 5'-GTG CAG GGT CCG AGG T-3'. U6: forward, 5'-TGC GGG TGC TCG CTT CGG CAG C-3', reverse, $5^{\prime}$-GTG CAG GGT CCG AGG $\mathrm{T}-3^{\prime}$. These used sequences of primers were bought from GeneScript (Nanjing, China). Finally, the relative level of miRNAs and mRNAs was calculated according to the $2^{-\Delta \Delta \mathrm{Ct}}$ methods. The experiments were repeated for three times independently, and 3-well was prepared for each sample.

\section{Western blot}

The supernatant, from lysed cancer cells or tissues, was used for determining the protein expression in this study using radio-immunoprecipitation assay (RIPA) lysate (Thermo Fisher Scientific). The lysates were boiled at $98^{\circ} \mathrm{C}$ for $10 \mathrm{~min}$. Then, $60 \mu \mathrm{g}$ proteins were added on sodium dodecyl sulfate (SDS)-polyacrylamide gel electrophoresis (SDS-PAGE) (Sangon). Afterward, these gels were transferred to $0.22 \mathrm{~nm}$ polyvinylidene difluoride (PVDF) membranes for $60 \mathrm{~min}$ (Bio-Rad, Hercules, CA, USA). Then, PVDF membranes were blocked with $2 \%$ phosphate buffer saline (PBS)-diluted bovine serum albumin (BSA) were incubated with the primary antibody at $4{ }^{\circ} \mathrm{C}$ for $8 \mathrm{~h}$. The protein bands were captured by using Tanon 4600SF system (Tanon, Shanghai, China) after coating with horseradish peroxidase (HRP)-conjugated goat-anti-mouse or goat anti-rabbit secondary antibody (Thermo Fisher Scientific). The anti-KLF7 and anti- $\beta$-actin were bought from Bioworld Technology (Nanjing, China). The experiments were carried out for three times independently.

\section{Cell counting kit-8 (CCK-8) assay}

In our study, the cell proliferation of LN-229 or U251 was assessed by performing CCK- 8 assay (Sigma). Firstly, $3 \times 10^{3}$ cells/well was prepared in 96-well plates (Corning, Shanghai, China). After $48 \mathrm{~h}$ transfection with miRNAs or plasmids, the OD value was measured in the microplate reader (Thermo Fisher Scientific). The experiments were conducted for three times independently, and 5-well was prepared for each group.

\section{Colony formation assay}

Firstly, 2000 cells/well was plated into a 6-well plate (Corning). After $48 \mathrm{~h}$ transfection with miRNAs or plasmids, the cells (LN-229 or U251) were maintained in normal fresh media. Next, the media was replaced every 3 days. Post 14 days incubation, the visible colonies were stained with $0.1 \%$ crystal violet (Sigma) after fixation with methanol for $1 \mathrm{~h}$, and they were captured using a microscopy (Leica, WETZLAR, German). The experiments were conducted for three times independently, and 5-well was prepared for each group.

\section{Trans-well assay}

Here, we put $1.0 \times 10^{5}$ cells per well into the topchambers of multi-well plates (Corning). Then, we added 10\% FBS-contained DMEM into the down chambers. Post $16 \mathrm{~h}$ incubation, the migrated cells were stained with crystal violet after fixation with $4 \%$ paraformaldehyde. In the end, we observed the migrated cells with a microscopy under $\times 200$ magnification (Leica). The experiments were carried out for three times independently, and 5-well was prepared for each group.

\section{Annexin-V/fluorescein isothiocyanate (FITC) and propidium iodide (PI) staining}

The cancer cells were seeded in 24-well plates, and they were harvested at $80 \mathrm{~g}$ for $5 \mathrm{~min}$ post $48 \mathrm{~h}$ transfection. Afterward, the LN-229 or U251 was stained with an Annexin V-FITC/PI kit (Solaribio, Beijing, China) in the dark following the manufacturer's instruction. Finally, the cell apoptosis rate of cancer cells was determined on Bio-Rad S3e flow cytometry (Hercules, CA, USA). The experiments were conducted for three times independently, and 3-well was prepared for each sample.

\section{Dual-luciferase reporter assay}

In this study, $3 \times 10^{4}$ cells (LN-229 or U251) was seeded in 24-well plates. The cells were co-transfected with pmirGLO-KLF7-3' UTR-WT (wide type), pmirGLOKLF7-3' UTR-mut (mutant), and miR-NC (negative control), miR-136-3p mimic, and anti-miR-136-3p. Post $48 \mathrm{~h}$ transfection, we analyzed the luciferase activity using dual-luciferase assay kit (Promega, Madison, USA). The experiments were performed for three times independently, and 5-well was prepared for each sample.

\section{Animal experiments}

The LN-229 cells were collected, and $6 \times 10^{6}$ cells were planted into 5 -week-old BALB/c nude mice. The mice were bought from the Model Animal Research Center of Nanjing University (Nanjing, China). At the initiation of tumor growth (on the third day), the lenti-viruses were injected into tumors, and this injection was repeated every 6 days. These animal experiments were approved by the ethics committee of Shandong Provincial Lanling People's Hospital. Afterward, the tumor volume was measured using the formula (length $\times$ width $\left.^{2}\right) / 2$ at the indicated time. Finally, the tumors were captured and weighted. 

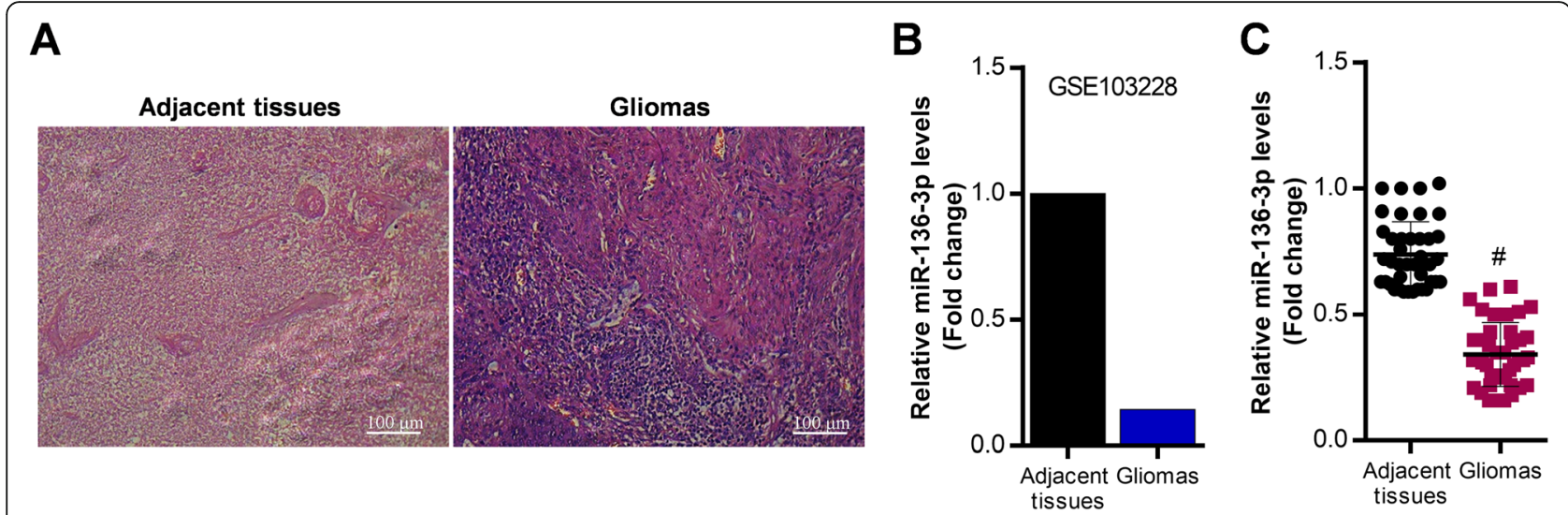

Fig. 1 MiR-136-3p is up-regulated in glioma tissues. a H \& E staining of pathologic alterations of glioma tissues. b GEO2R analysis of miR-136-3p in microarray GSE103228. c qRT-PCR analysis of miR-136-3p in adjacent tissues and in glioma tissues; \#p < 0.01, compared with adjacent tissues

\section{Data analysis}

The data here were indicated as the mean \pm standard deviation (S.D.). A $p$ value $<0.05$ was statistically significant. All the showed data were analyzed statistically using GraphPad Prism 6 (GraphPad Software, CA, USA). The statistical significance was examined by Two-tailed Student's $t$ test for two groups comparisons and one-way analysis of variance (ANOVA) test with post hoc analysis contrasts for multi-groups comparisons.

\section{Results}

MiR-136-3p is elevated in glioma tissues

To investigate the role of miR-136-3p in glioma development, we collected tumors from patients with glioma in the hospital. The clinical characteristics of 41 patients were showed in Table 1 . The H\&E staining showed that glioma tissues showed significant pathologic alterations compared with adjacent tissues (Fig. 1a). GEO2R analysis and qRT-PCR analysis revealed that miR-136-3p was significantly decreased in glioma tissues compared with adjacent tissues (Fig. 1b, c).

Table 1 Clinical characteristics of 41 patients

\begin{tabular}{|c|c|c|c|c|c|}
\hline \multirow{2}{*}{$\begin{array}{l}\text { Clinicopathologic } \\
\text { feature }\end{array}$} & \multirow[t]{2}{*}{$\boldsymbol{n}(\%)$} & \multicolumn{3}{|c|}{ miR-136-3p } & \multirow[t]{2}{*}{$\boldsymbol{p}$ value } \\
\hline & & High & Low & mean \pm SEM & \\
\hline Age & & & & & $p=0.3603$ \\
\hline$\leq 40$ & 19 (46.34\%) & 10 & 9 & $1.89 \pm 0.18$ & \\
\hline$\geq 40$ & $22(53.66 \%)$ & 10 & 12 & $1.92 \pm 0.17$ & \\
\hline Gender & & & & & $p=0.2104$ \\
\hline Female & $18(43.90 \%)$ & 8 & 10 & $1.91 \pm 0.19$ & \\
\hline Male & $24(56.10 \%)$ & 13 & 11 & $1.79 \pm 0.18$ & \\
\hline Surgery & & & & & $p=0.4472$ \\
\hline Biopsy & $7(17.07 \%)$ & 4 & 3 & $2.03 \pm 0.20$ & \\
\hline Partial resection & $14(34.15 \%)$ & 5 & 9 & $2.15 \pm 0.18$ & \\
\hline Gross total resection & $20(48.78 \%)$ & 9 & 11 & $2.07 \pm 0.21$ & \\
\hline WHO grade (\%) & & & & & $p<0.0001$ \\
\hline$\|$ & $8(30.00 \%)$ & 2 & 6 & $2.91 \pm 0.26$ & \\
\hline III & $12(33.33 \%)$ & 4 & 8 & $2.05 \pm 0.21$ & \\
\hline IV & $21(36.67 \%)$ & 5 & 17 & $1.68 \pm 0.17$ & \\
\hline Low expression & $18(43.90 \%)$ & 13 & 5 & $4.19 \pm 0.41$ & $p<0.0001$ \\
\hline High expression & $23(56.10 \%)$ & 4 & 19 & $1.98 \pm 0.22$ & \\
\hline Tumor size & & & & & $p=0.3514$ \\
\hline$<4 \mathrm{~cm}$ & $16(39.02 \%)$ & 7 & 8 & $2.18 \pm 0.21$ & \\
\hline$\geq 4 \mathrm{~cm}$ & 25 (60.98\%) & 11 & 14 & $2.24 \pm 0.23$ & \\
\hline
\end{tabular}




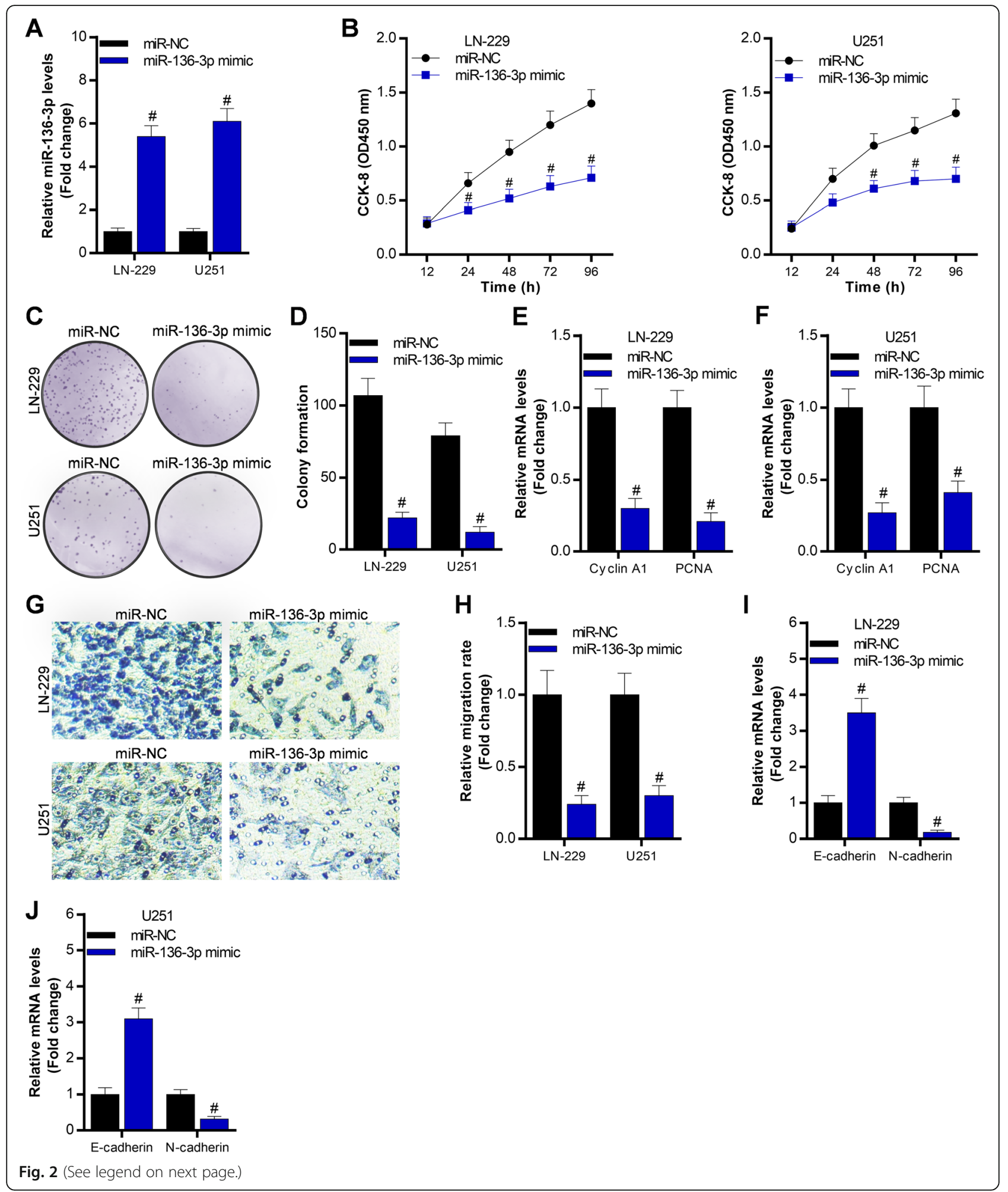


(See figure on previous page.)

Fig. 2 Overexpression of miR-136-3p inhibits glioma cell proliferation and migration in LN-229 and U251 cells. a qRT-PCR analysis of miR-136-3p in LN-229 and U251 cells post $48 \mathrm{~h}$ transfection with miR-NC or miR-136-3p mimic; \#p < 0.01, compared with miR-NC. $\mathbf{b}$ CCK-8 assay determination of proliferation of LN-229 and U251 cells after transfection with miR-NC or miR-136-3p mimic at the indicated time; \#p < 0.01, compared with miR-NC. $\mathbf{c}$ d Soft-agar colony formation assay analysis of glioma cell growth after 15 days transfection with miR-NC or miR-136-3p mimic; $\# p<0.01$, compared with miR-NC. e, f qRT-PCR analysis of cell growth-associated genes, including Cyclin A1 and PCNA, in LN-229 and U251 cells after $48 \mathrm{~h}$ transfection with miR-NC or miR-136-3p mimic; $\# p<0.01$, compared with miR-NC. $\mathbf{g}$, $\mathbf{h}$ Trans-well assay analysis of migration ability of transfected LN-229 and U251 cells with miR-NC or miR-136-3p mimic; \#p < 0.01, compared with miR-NC. $\mathbf{i}$, $\mathbf{j}$ qRT-PCR analysis of cell migration-associated genes, including E-cadherin and N-cadherin, in LN-229 and U251 cells after $48 \mathrm{~h}$ transfection with miR-NC or miR-136-3p mimic; \#p < 0.01, compared with miR-NC

\section{Overexpression of miR-136-3p suppresses glioma cell growth and migration}

Here, we overexpressed miR-136-3p in LN-229 and U251 to detect the glioma cell growth and migration. The data showed that miR-136-3p mimics efficiently overexpressed miR-136-3p in LN-229 and U251 cells (Fig. 2a). The CCK-8 assay told that overexpression of miR-136-3p significantly inhibited glioma cell proliferation (Fig. 2b). Soft-agar colony formation assay analysis demonstrated that overexpression of miR-136-3p obviously repressed glioma cell colony formation of LN-229 and U251 cells (Fig. 2c, d). Meanwhile, overexpression of miR-136-3p significantly downregulated cell growthassociated gene expression, including Cyclin A1 and PCNA in LN-229 and U251 cells (Fig. 2e, f). Finally, trans-well assay demonstrated that overexpression of
miR-136-3p markedly decreased the migration of LN229 and U251 (Fig. 2g, h). Finally, we found that overexpression of miR-136-3p elevated expression of migration-associated gene E-cadherin, and it reduced $\mathrm{N}$ cadherin levels in LN-229 and U251.

\section{Overexpression of miR-136-3p promotes glioma cell apoptosis}

To determine the effect of miR-136-3p on glioma cell apoptosis, we conducted annexin V-FITC/PI staining. The data exhibited that overexpression of miR-136-3p significantly promotes apoptosis of LN-229 and U251 cells (Fig. 3a, b). Consistently, qRT-PCR demonstrated that overexpression of miR-136-3p elevated Bax expression, and inhibited Bcl-2 expression in $\mathrm{LN}-229$ and U251 cells (Fig. 3c).

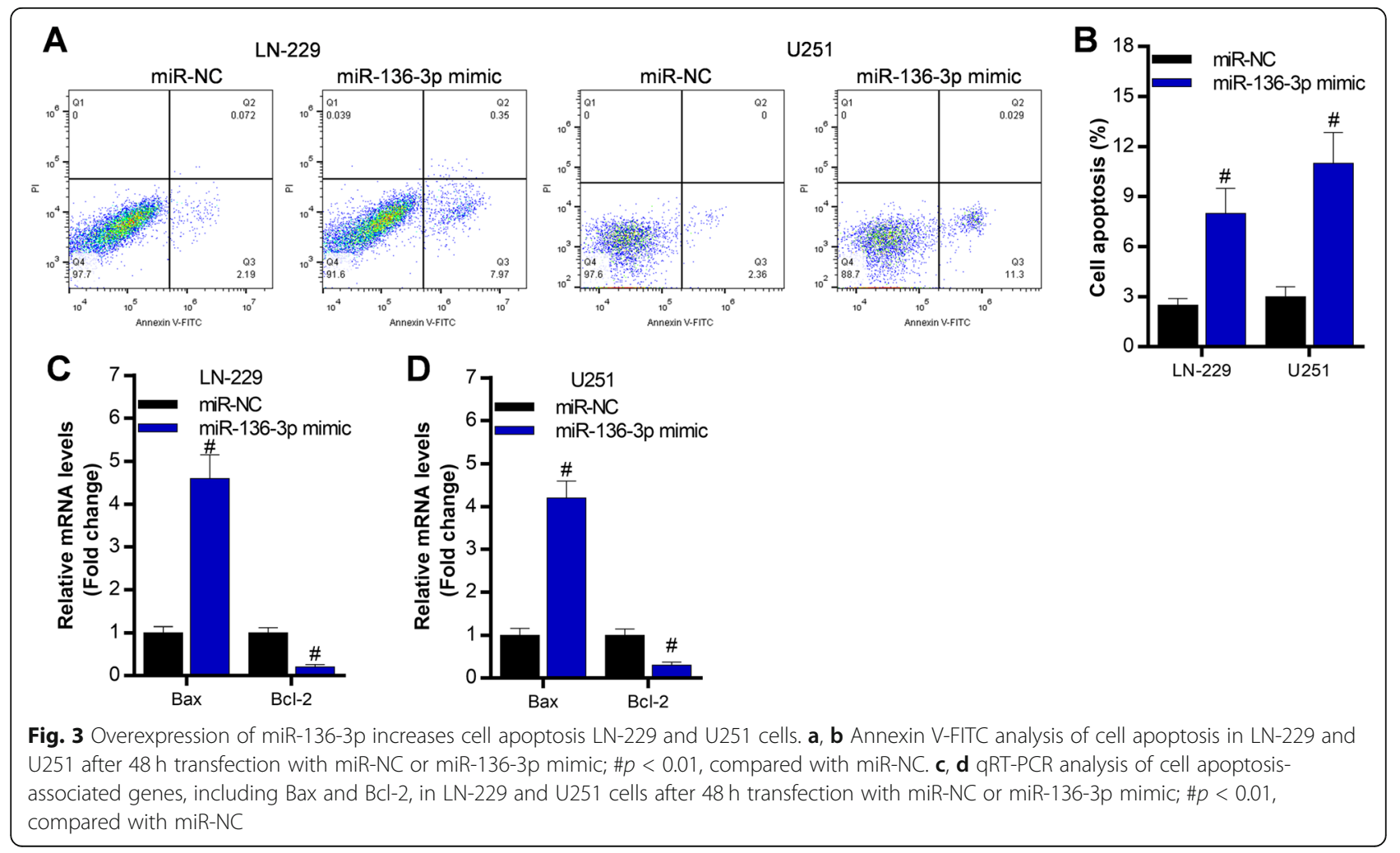


KLF7 is a direct target of miR-136-3p in glioma cells To investigate the potential molecular mechanism of miR-136$3 p$ in gliomas, we predicted the downstream targets of miR-136$3 \mathrm{p}$ using TargetScan tools. Base on comprehensive consideration of both the bioinformatic prediction and the previous studies [19-22], we can conclude that KLF7 is an oncogene in glioma or other cancers. Thus, we selected KLF as the target of miR-136$3 p$ in glioma (Fig. 4a). Luciferase reporter assay expressed that overexpression of miR-136-3p significantly inhibited luciferase activity of KLF7 3' UTR, which was upregulated by knocking miR136-3p down in LN-229 and U251 (Fig. 4c, d). Then, qRT-PCR analysis and western blot analysis revealed that miR-136-3p negatively modulated KLF7 expression (Fig. 4e, f, g). Moreover, the miR-136-3p expression is negatively associated with KLF7 levels in clinical samples (Fig. 4h). Additionally, KLF7 was increased in glioma tissues (Fig. 4i).

\section{Overexpression of KLF7 partly reverses miR-136-3p-} controlled glioma cell growth, migration, and apoptosis in vitro and in vivo

To determine whether KLF7 is necessarily required for function of miR-136-3p in glioma development, we co-overexpressed

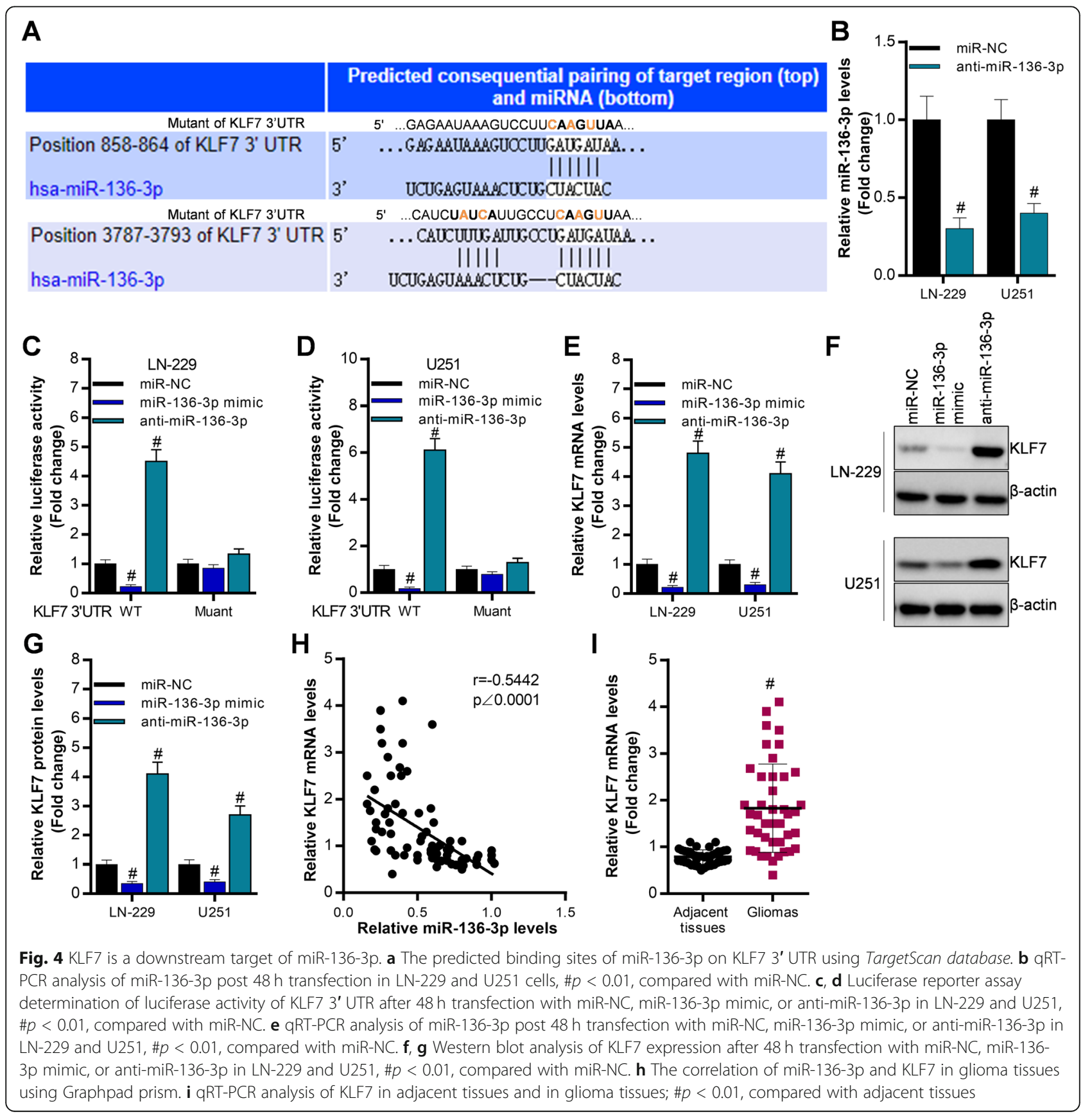




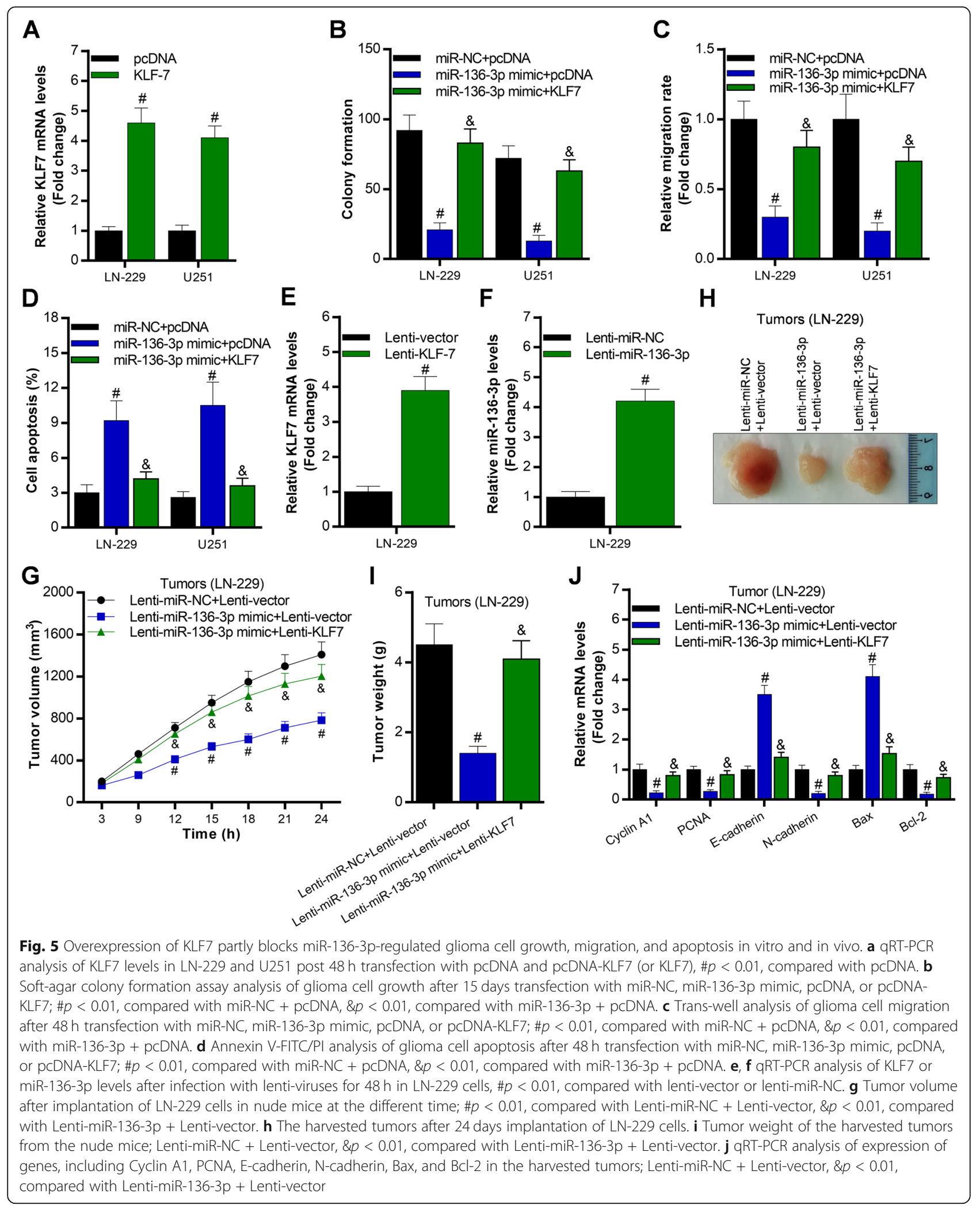


KLF7 and miR-136-3p in LN-229 and U251. The data showed KLF7 was successfully overexpressed by transfecting with the full length of KLF7 complementary DNA-contained plasmid (Fig. 5a). Firstly, we found that overexpression of KLF7 partly blocked miR-136-3p-regulated glioma cell growth, migration, and apoptosis of LN-229 and U251 cells (Fig. 5b, c, d). Furthermore, we also co-overexpressed KLF7 and miR-136-3p in vivo using lenti-viruses (Fig. 5e, f). Overexpression of miR-136-3p significantly decreased tumor growth indicated by tumor volume and tumor weight in nude mice (Fig. 5g, h, i). Meanwhile, overexpression of miR-136-3p obviously downregulated expression of genes, including Cyclin A1, PCNA, N-cadherin, and $\mathrm{Bcl}-2$, and miR-136-3p overexpression significantly upregulated E-cadherin and Bax expression in tumors (Fig. 5j). Interestingly, we found that overexpression of KLF7 partly blocked miR-1363p-induced suppression of tumor growth by regulating Cyclin A1, PCNA, E-cadherin, N-cadherin, Bax, and Bcl-2 (Fig. 5g- j).

\section{Discussion}

Glioma is the most malignant tumor in brain tissues, and it has been characterized by fast infiltrative growth and dysregulated metastasis resulting in a poor overall survival rate for glioma patients [23]. Therefore, to explore molecular mechanisms could offer novel therapeutic targets for glioma treatment. Up to now, it is difficult to accurately predict prognosis for glioma patients [23]. Therefore, it is of great significance to find novel and invaluable prognostic biomarkers for glioma patients. Nowadays, increasing studies have proved that a series of miRNAs are involved in progression of multiple styles of tumors [7-13]. For instance, miR-662 [6], miR-532-5p [14], miR-199a [15], miR-940 [24], miR200b [17], miR-34a [18], miR-130b [19], and miR-148a [20] all play an essential role in gliomas. Herein, we investigated the role of miR-136-3p and its target gene KLF7 in glioma development.

MiR-136-3p has been reported to inhibit progression of thyroid cancer [16], implying that miR-136-3p may serve as a tumor suppressor in tumors. However, little is known about the functions of miR-136-3p in other cancers, including gliomas. In this study, miR136-3p was decreased in glioma tissues compared with the adjacent tissues. Importantly, the ability of cell growth and migration was inhibited in miR-1363p-overexpressed cancer cells (LN-229 and U251), and the cell apoptosis was promoted in miR-136-3poverexpressed cancer cells, suggesting that miR-136$3 p$ may function as a tumor suppressor in gliomas. Consistently, overexpression of miR-136-3p significantly inhibited tumor growth in nude mice, conforming that miR-136-3p can serve as a cancer suppressor and it can inhibit the tumorigenesis of glioma.
MiRNAs show its function mainly by targeting 3' UTR of mRNA in many diseases [7-9]. We predicted the target genes of miR-136-3p via using TargetScan database. Our results demonstrated that KLF7 was a direct target of miR-136-3p. Previous studies have revealed that KLF7 can promote polyamine biosynthesis and glioma development by activating argininosuccinate lyase [21], and meanwhile, KLF7 can be modulated by Linc00669/miR-193a axis and it can advance non-small cell lung cancer [20], suggesting that KLF7 is an oncogene. In this study, we observed that KLF7 expression was inhibited by overexpression of miR136-3p, and KLF7 levels was increased by knockdown of miR-136-3p in cancer cells, indicating that KLF7 was a direct target of miR-136-3p in glioma. Then, in vitro and in vivo experiments were performed to determine whether KLF7 was required for miR-136$3 p$ exerting its functions in glioma. The data showed that overexpression of KLF7 can partly block overexpression of miR-136-3p-mediated suppression of glioma progression by regulating cell growth, migration, and apoptosis of cancer cells.

\section{Conclusion}

These findings demonstrated that miR-136-3p is significantly decreased in cancer tissues and it serves as a tumor suppressor by directly targeting KLF7 in gliomas. The results also encourage that miR-136-3p and KLF7 might be promising novel biomarkers and therapeutic targets for glioma patients' treatment.

\section{Acknowledgements \\ We thank our team for technical cooperation.}

\section{Author's contributions}

Yanwu Xu designed the project, performed the animal experiments, and cultured the cell lines and conducted the cell experiments. He analyzed the experimental data. He wrote the manuscript including discussion and suggestions. He also provided the financial support. The author(s) read and approved the final manuscript.

\section{Funding}

None.

\section{Availability of data and materials}

The data and materials of this manuscript would be provided if we are required.

\section{Ethics approval and consent to participate}

All applicable international, national, and/or institutional guidelines for the care and use of animals were followed. All procedures performed in our studies involving human participants were in accordance with the ethical standards of the institutional and/or national research committee and with the 1964 Helsinki Declaration and its later amendments or comparable ethical standards.

Informed consent was obtained from all included participants.

Competing interests

All authors declare no competing financial interests. 
Received: 16 February 2020 Accepted: 7 July 2020

Published online: 16 July 2020

\section{References}

1. Visser O, Ardanaz E, Botta L, Sant M, Tavilla A, Minicozzi P, Group E-W. Survival of adults with primary malignant brain tumours in Europe; results of the EUROCARE-5 study. Eur. J. Cancer. 2015;51(15):2231-41.

2. Lapointe S, Perry A, Butowski NA. Primary brain tumours in adults. Lancet. 2018:392(10145):432-46

3. McNamara S. Treatment of primary brain tumours in adults. Nurs. Stand. 2012;27(14):42-7.

4. Wang Q, Zhang J, Liu Y, Zhang W, Zhou J, Duan R, Pu P, Kang C, Han L. A novel cell cycle-associated IncRNA, HOXA11-AS, is transcribed from the 5prime end of the HOXA transcript and is a biomarker of progression in glioma. Cancer Lett. 2016:373(2):251-9.

5. Hattermann K, Fluh C, Engel D, Mehdorn HM, Synowitz M, Mentlein R, HeldFeindt J. Stem cell markers in glioma progression and recurrence. Int. J. Oncol. 2016;49(5):1899-910.

6. Song $\mathrm{Q}$, Pang H, Qi L, Liang C, Wang T, Wang W, Li R. Low microRNA-622 expression predicts poor prognosis and is associated with ZEB2 in glioma. Onco Targets Ther. 2019;12:7387-97.

7. Callegari E, D'Abundo L, Guerriero P, Simioni C, Elamin BK, Russo M, Cani A, Bassi C, Zagatti B, Giacomelli L, Blandamura S, Moshiri F, Ultimo S, Frassoldati A, Altavilla G, Gramantieri L, Neri LM, Sabbioni S, Negrini M. miR199a-3p modulates MTOR and PAK4 pathways and inhibits tumor growth in a hepatocellular carcinoma transgenic mouse model. Molecular therapy. Nucleic acids. 2018;11:485-93.

8. Cai $Y, Y u X$, Hu S, Yu J. A brief review on the mechanisms of miRNA regulation. Genomics, proteomics \& bioinformatics. 2009;7(4):147-54.

9. Ni WJ, Leng XM. miRNA-dependent activation of mRNA translation. MicroRNA. 2016;5(2):83-6.

10. Mendell JT. MicroRNAs: critical regulators of development, cellular physiology and malignancy. Cell cycle. 2005;4(9):1179-84.

11. Abe M, Bonini NM. MicroRNAs and neurodegeneration: role and impact. Trends Cell Biol. 2013;23(1):30-6.

12. Mao B, Wang G. MicroRNAs involved with hepatocellular carcinoma (Review). Oncol. Rep. 2015;34(6):2811-20.

13. Wang $S$, Yin Y, Liu S. Roles of microRNAs during glioma tumorigenesis and progression. Histol. Histopathol. 2019;34(3):213-22.

14. Wang YP, Liu J, Liu D, Wang XD, Bian AM, Fang DZ, Hui XB. MiR-532-5p acts as a tumor suppressor and inhibits glioma cell proliferation by targeting CSF1. Eur. Rev. Med. Pharmacol. Sci. 2019:23(20):8964-70.

15. Li W, Wang L, Ji XB, Wang LH, Ge X, Liu WT, Chen L, Zheng Z, Shi ZM, Liu LZ, Lin MC, Chen JY, Jiang BH. MiR-199a inhibits tumor growth and attenuates chemoresistance by targeting K-RAS via AKT and ERK signalings. Front. Oncol. 2019:9:1071

16. Xiong Y, Kotian S, Zeiger MA, Zhang L, Kebebew E. miR-126-3p inhibits thyroid cancer cell growth and metastasis, and is associated with aggressive thyroid cancer. PLoS One. 2015; 10(8): e0130496.

17. Yang Y, Wu J, Guan H, Cai J, Fang L, Li J, Li M. MiR-136 promotes apoptosis of glioma cells by targeting AEG-1 and BCl-2. FEBS Lett. 2012;586(20):360812

18. Yao J, Zhang H, Liu C, Chen S, Qian R, Zhao K. miR-450b-3p inhibited the proliferation of gastric cancer via regulating KLF7. Cancer Cell Int. 2020; 20 47.

19. Zhao L, Zhang Y, Liu J, Yin W, Jin D, Wang D, Zhang W. miR-185 inhibits the proliferation and invasion of non-small cell lung cancer by targeting KLF7. Oncol. Res. 2019;27(9):1015-23.

20. An YX, Shang YJ, Xu ZW, Zhang QC, Wang Z, Xuan WX, Zhang XJ. STAT3induced long noncoding RNA LINC00668 promotes migration and invasion of non-small cell lung cancer via the miR-193a/KLF7 axis. Biomed. Pharmacother. 2019;116:109023.

21. Guan F, Kang Z, Zhang JT, Xue NN, Yin H, Wang L, Mao BB, Peng WC, Zhang BL, Liang X, Hu ZQ. KLF7 promotes polyamine biosynthesis and glioma development through transcriptionally activating ASL. Biochem. Biophys. Res. Commun. 2019:514(1):51-7.

22. Jiang Z, Yu T, Fan Z, Yang H, Lin X. Kruppel-like factor 7 is a marker of aggressive gastric cancer and poor prognosis. Cell. Physiol. Biochem. 2017; 43(3):1090-9.

23. Beyer $\mathrm{S}$, von Bueren $\mathrm{AO}$, Klautke $\mathrm{G}$, Guckenberger M, Kortmann RD, Pietschmann S, Muller K. A systematic review on the characteristics, treatments and outcomes of the patients with primary spinal glioblastomas or gliosarcomas reported in literature until March 2015. PLoS One. 2016; 11(2):e0148312.

24. Luo H, Xu R, Chen B, Dong S, Zhou F, Yu T, Xu G, Zhang J, Wang Y, You Y. MicroRNA-940 inhibits glioma cells proliferation and cell cycle progression by targeting CKS1. American journal of translational research. 2019;11(8): 4851-65.

\section{Publisher's Note}

Springer Nature remains neutral with regard to jurisdictional claims in published maps and institutional affiliations.
Ready to submit your research? Choose BMC and benefit from:

- fast, convenient online submission

- thorough peer review by experienced researchers in your field

- rapid publication on acceptance

- support for research data, including large and complex data types

- gold Open Access which fosters wider collaboration and increased citations

- maximum visibility for your research: over $100 \mathrm{M}$ website views per year

At BMC, research is always in progress.

Learn more biomedcentral.com/submissions 